\title{
EFFECT OF ULTRASONIC SCALING ON MICROLEAKAGE OF CLASS V COMPOSITE RESTORATIONS BONDED WITH DIFFERENT ADHESIVES
}

\author{
Ashraf Ibrahim Ali Ibrahim*, Tayseer Mohamed Maaly** and Enas Tawfik Enan***
}

\begin{abstract}
Objectives: The current study was conducted to investigate the influence of ultrasonic scaling on microleakage under composite restorations bonded with 3 different adhesives.

Methods: Sixty standerdized class V cavities were prepared in extracted human molars that were divided in to three groups, according to the type of applied adhesive, as follows: Group A: Fuji Bond LC, Glass ionomer (GI) adhesive, Group B: Clearfill S3 Bond, Self-Etch (SE) adhesive and Group C: OptiBond FL, Etch and Rinse (ER) adhesive. After cavity preparation and composite filling, teeth of each group were further divided into scaled and un-scaled subgroups. Teeth were sectioned buccolingually and the gingival margins of the teeth were evaluated and scored for dye penetration to check the microleakage.
\end{abstract}

Results: Scaling caused a significant increase in microleakage scores of SE adhesive. On the other hand, both ER adhesive and GI adhesive showed insignificant change in microleakage scores after exposure to the ultrasonic scaling.Self- Etch adhesive showed the highest mean rank microleakage value (13.7), while GI adhesive had the lowest mean rank microleakage value (12.9) in the scaled group. Significant difference between the scaled (mean rank 13.7) and un-scaled (mean rank 7.3) subgroups was found only with SE adhesive $(\mathrm{p}=0.007)$. On the other hand, insignificant differences $(p=0.06)$ were noticed between the two subgroups of GI and ER adhesive.

Conclusion: Ultrasonic scaling caused significant increase in microleakage of self-etch adhesive bonded restorations. While GI and ER adhevies bonded restorations revealed more resistance to the effect of ultrasonic scaling.

KEY-WORDS: Ultrasonic scaling; Microleakage; Glass ionomer; Self-Etch; Adhesive.

\footnotetext{
* Associate Professor, Department of Operative Dentistry, Faculty of Dentistry, Mansoura University, Mansoura, Egypt. ** Lecturer, Department of Dental Biomaterials, Faculty of Dentistry, Zagazig university

*** Associate Professor, Department of Dental Biomaterials, Faculty of Dentistry, Mansoura University, Mansoura, Egypt.
} 


\section{INTRODUCTION}

Preserving marginal integrity of dental restorations is a challenge for dentists. Bond failure and loss of marginal seal may lead to microleakage, with subsequent possibility of marginal discoloration, postoperative sensitivity, recurrent caries or even restorative failure. ${ }^{1-4}$

Adhesives play an essential role in preventing microleakage at the composite/tooth interface. Therefore, different adhesive systems have been proposed by manufacturers, depending on different adhesion strategies. ${ }^{5}$ Traditionally, bonding to dental tissues was obtained by etch and rinse (ER) strategy, where phosphoric acid was applied for etching, followed by rinsing and applying an adhesive agent. ${ }^{6}$ Later on, more simple products were introduced with the development of self-etching (SE) adhesives, eliminating the need for conditioning, rinsing and drying steps that were critical for the adhesion protocol.${ }^{6,7}$ Nowadays, three main adhesive systems are available in the markets; the Etch and Rinse (ER) (either in three or two clinical steps), the Self-Etch (SE) adhesive systems (available in two or one clinical step (s), and the glass ionomer (GI) adhesives. ${ }^{8,9}$ The glass-ionomer based adhesive was presented to the markets by 1995 as a diluted form of resin-modified glass-ionomer cement (GIC). It was reported to have high bond strength and high success rate in retaining composite restorations to cervical cavities. ${ }^{10,11}$ De-bonding and microleakage can be observed when composite restorations are submitted to stresses during or after polymerization. If the generated stresses exceed the bond strength between the dental substrate and adhesive system, a contraction gap will be formed, jeopardizing the restoration's longevity., 12, 13 During dental hygiene procedures, such as ultrasonic scaling, mechanical stimulation may create stresses at the compositetooth interface, particularly at the gingival margins. These stresses can affect the tooth-restoration bond..$^{14}$
Although some previous studies ${ }^{5-8}$ compared different resin adhesive systems, yet, none of them included a comparison with glass-ionomer adhesives, especially under stresses of ultrasonic scaling. Thus, the current study aimed to evaluate the effect of ultrasonic scaling, in presence of different adhesive systems, on microleakage of class $\mathrm{V}$ composite restorations. The null hypothesis tested was that the application of ultrasonic scaling would have no effect on microleakage around resin composite restorations.

\section{MATERIALS AND METHODS}

\section{Specimens preparation and grouping}

Thirty freshly extracted human permanent maxillary and mandibular molars, free from caries, cracks and restorations, were selected for this study. The study was approved by the Ethics Committee of Mansoura University, Egypt. The selected teeth were disinfected for 24 hours in $2 \%$ sodium azide solution, and then stored in normal saline at $37^{\circ} \mathrm{C}$ \pm 1 temperature until performing the test steps. Standardized Class V cavities were prepared on both the buccal and lingual surfaces of the collected teeth, giving rise to 60 cavity specimens $(n=60)$. Each cavity was prepared $1 \mathrm{~mm}$ apical to cementoenamel junction with dimensions of $1.5 \mathrm{~mm}$ depth, 4 $\mathrm{mm}$ mesio-distal width and $2 \mathrm{~mm}$ occluso-cervical height, using a 557 carbide bur. ${ }^{15}$

The collected teeth were divided into three equal groups (10 teeth each) according to the type of applied adhesive system, as follows:

Group A: Fuji Bond LC ${ }^{\circledR}$ (GC, Tokyo, Japan), Glass ionomer (GI) adhesive.

Group B: Clearfill S3 Bond ${ }^{\circledR}$ (Kuraray, Tokyo, Japan), Self-Etch (SE) adhesive.

Group C: OptiBond $\mathrm{FL}^{\circledR}$ (Kerr, Italy), Etch and Rinse (ER) adhesive.

In a later stage, after application of resin composite restorations, one surface of each tooth 


\section{Restorative procedure}

Each adhesive was applied according to its manufacturers' instructions. For group A, Fuji bond LC conditioner was firstly applied to dentin surface for 10 seconds, then washed and dried. After that, the powder/liquid adhesive was mixed according to manufacturer instructions, then applied and cured for 20 seconds. For group B, Clearfill S3 adhesive was directly applied to the cavity walls and left undisturbed for 20 seconds, then air thinned for 5 seconds and light cured for 10 seconds. For group C, 37\% phosphoric acid etchant was firstly applied to enamel and dentine surfaces for 30 and 15 seconds, respectively, then washed and dried. Optibond FL primer was then applied to dentin surface for 15 seconds and air dried for 5 seconds. Then the adhesive was applied to dentin surface for 15 seconds, air thinned for 3 seconds and light cured for 20 seconds. All bonding systems were light cured using a LED light-curing unit (LEDition, IvoclarVivadent, Germany) with light intensity of $600 \mathrm{~mW} / \mathrm{cm}^{2}$.

After application of bonding agents, all cavities were filled with a nano-filled resin composite (Filtek Z-350 XT, 3M ESPE, St. Paul, MN, USA) in two increments. Each increment was light cured separately for 40 seconds using the same curing unit used with adhesives. After curing and removal of excess material, the restorations were polished using polishing discs(Sof-Lex, 3M ESPE) from rough to fine to ensure well-polished surfaces.

\section{Scaling procedure}

Ultrasonic scaling was performed with WOODPEKER UDS-J Ultrasonic Scaler (Information Industrial Park, Guilin National High-Tech Zone, Guilin, Guangxi, 541004 P.R.China) using G2 tip, under copious water flow at level 2 power setting. The scaling tip was angled approximately to $15^{\circ}$ to the restoration surface. The lateral side of the tip was placed in contact with the composite-tooth margin, and the margin was traced for 60 seconds. All specimens were scaled by the same operator who applied moderate hand pressure. To simulate the clinical conditions, specimens were subjected to thermocycling in water baths with 2500 cycles alternatively at $5^{\circ} \mathrm{C}$ and $55^{\circ} \mathrm{C}$ with a dwell time of 15 seconds.

\section{Microleakage Test}

For this test, the root apexes were sealed with molten blue inlay wax and all teeth surfaces were covered with two coats of nail varnish, leaving an uncoated area including the restoration plus $1 \mathrm{~mm}$ of surrounding tooth surface. The coated teeth were then immersed in $5 \mathrm{wt} \%$ Fuchsine solution (Fischer ScientificCompany, Fairlawn, NJ, USA) for $24 \mathrm{~h}$ at $37^{\circ} \mathrm{C}$, then rinsed with water. Teeth were embedded in Clear chemical cure acrylic resin and then cut longitudinally with a dental sectioning disc (Vision flex diamond disc, Brasseler). The sections were examined under a digital microscope (VHX 600, Keyence) at 30X magnification power, and dye penetration was quantitatively measured with a built-in image analysis software. ${ }^{15}$ The gingival margins of the teeth were evaluated and scored for dye penetration from 0 to 3 as follows. ${ }^{16}$

0: no dye penetration.

1: Dye penetration along the cavity wall, but less than half of cavity depth.

2: Dye penetration along the cavity wall, more than half of the cavity depth.

3: Dye penetration spreading to and along the axial wall.

\section{Statistical analysis}

The collected data were assessed using statistical analysis software (SPSS 12.0, SPSS, Chicago, Illinois). Due to the non-normal distribution of data, non-parametric statistical tests were performed. Statistical differences were analyzed with 
Chi-Square, Mann-Whitney and Kruskal-Wallis tests, and the level of significance was set at $\alpha=0.05$.

\section{RESULTS}

Microleakage score results of the tested groups were tabulated and presented in Tables 1 through Table 4. Representative sections of microleakage scores were shown in (Figure 1). Table 1 shows the Kruskal-Wallis results comparing scores of the three types of adhesives under each type of treatment, unscaled and scaled. These results showed insignificant differences $(p>0.05)$ between the three types of tested adhesives, whether under the scaled or unscaled conditions. In (Table 2), a comparison of the scaled to un-scaled specimens under each type of adhesive was shown separately using Chi-Square test. The results revealed insignificant differences ( $p>0.05$ ) among both Fuji Bond Lc and Optibond adhesives, while Clearfill S3 scores showed significant differences $(p=0.02)$ between the scaled and un-scaled specimens under microleakage score 0 . To compare mean ranks of the three types of adhesives, Kruskal-Wallis test was applied, and results were presented in (Table 3). It could be noticed that Fuji Bond Lc had the highest mean rank value (8.10), while Clearfill S3 had the lowest value (7.30) under un-scaled group. However, the differences were insignificant between the three types of adhesives, whether for the un-scaled $(\mathrm{p}=0.217)$ or the scaled $(\mathrm{p}=0.251)$ subgroups. To compare mean ranks of the 2 subgroups -scaled and un-scaled- under each type of adhesives, MannWhitney test was used, and results were shown in (Table 4). From this table, a significant difference between the scaled and un-scaled subgroups was found only with Clearfill S3 adhesive ( $p=0.007)$. On the other hand, insignificant differences were noticed between the two subgroups of Fuji Bond Lc $(p=0.06)$ and also Optibond FL $(p=0.06)$.

TABLE (1): Comparison of microleakage scores among different groups.

\begin{tabular}{|c|c|c|c|c|c|c|c|}
\hline \multirow{2}{*}{\multicolumn{3}{|c|}{ Treatment/adhesives }} & \multicolumn{4}{|c|}{ Microleakage Scores } & \multirow{3}{*}{$\begin{array}{c}\text { Total } \\
10\end{array}$} \\
\hline & & & \multirow{2}{*}{$\begin{array}{l}\mathbf{0} \\
5\end{array}$} & \multirow{2}{*}{$\frac{1}{2}$} & \multirow{2}{*}{$\frac{2}{3}$} & \multirow{2}{*}{$\begin{array}{l}3 \\
0\end{array}$} & \\
\hline \multirow{7}{*}{ Un-scaled } & \multirow{2}{*}{ Fuji Bond Lc } & Count & & & & & \\
\hline & & $\%$ & $50.0 \%$ & $20.0 \%$ & $30.0 \%$ & $0.0 \%$ & $100 \%$ \\
\hline & \multirow{2}{*}{ Clearfill S3 } & Count & 8 & 2 & 0 & 0 & 10 \\
\hline & & $\%$ & $80.0 \%$ & $20.0 \%$ & $0.0 \%$ & $0.0 \%$ & $100.0 \%$ \\
\hline & \multirow{2}{*}{ Optibond } & Count & 5 & 4 & 1 & 0 & 10 \\
\hline & & $\%$ & $50.0 \%$ & $40.0 \%$ & $10.0 \%$ & $0.0 \%$ & $100.0 \%$ \\
\hline & \multicolumn{2}{|c|}{ Kruskal Wallis P value } & 0.07 & 0.06 & NA & NA & \\
\hline \multirow{7}{*}{ Scaled } & \multirow{2}{*}{ Fuji Bond Lc } & Count & 2 & 2 & 2 & 4 & 10 \\
\hline & & $\%$ & $20.0 \%$ & $20.0 \%$ & $20.0 \%$ & $40.0 \%$ & $100.0 \%$ \\
\hline & \multirow{2}{*}{ Clearfill S3 } & Count & 2 & 6 & 2 & 0 & 10 \\
\hline & & $\%$ & $20.0 \%$ & $60.0 \%$ & $20.0 \%$ & $0.0 \%$ & $100.0 \%$ \\
\hline & \multirow{2}{*}{ Optibond } & Count & 2 & 3 & 3 & 2 & 10 \\
\hline & & $\%$ & $20.0 \%$ & $30.0 \%$ & $30.0 \%$ & $20.0 \%$ & $100.0 \%$ \\
\hline & \multicolumn{2}{|c|}{ Kruskal Wallis P value } & 0.12 & 0.07 & 0.09 & 0.07 & \\
\hline
\end{tabular}

NA- Not applicable, 0: no dye penetration, 1: Dye penetration along the cavity wall, but less than half of cavity depth, 2: Dye penetration along the cavity wall, more than half of the cavity depth, 3: Dye penetration spreading to and along the axial wall. 
TABLE (2): Comparison of microleakage score between scaled and unscaled subgroups.

\begin{tabular}{|c|c|c|c|c|c|c|c|}
\hline \multirow{2}{*}{\multicolumn{2}{|c|}{ Adhesive/treatment }} & & \multicolumn{4}{|c|}{ Microleakage Scores } & \multirow{3}{*}{$\begin{array}{c}\text { Total } \\
10\end{array}$} \\
\hline & & & \multirow{2}{*}{$\begin{array}{l}\mathbf{0} \\
5\end{array}$} & \multirow{2}{*}{$\frac{1}{2}$} & \multirow{2}{*}{$\frac{2}{3}$} & \multirow{2}{*}{$\begin{array}{l}3 \\
0\end{array}$} & \\
\hline \multirow{5}{*}{ Fuji Bond Lc } & \multirow{2}{*}{ Un-scaled } & Count & & & & & \\
\hline & & $\%$ & $50.0 \%$ & $20.0 \%$ & $30.0 \%$ & $0.0 \%$ & $100.0 \%$ \\
\hline & \multirow{2}{*}{ Scaled } & Count & 2 & 2 & 2 & 4 & 10 \\
\hline & & $\%$ & $20.0 \%$ & $20.0 \%$ & $20.0 \%$ & $40.0 \%$ & $100.0 \%$ \\
\hline & \multicolumn{2}{|c|}{ Chi-Square, P- value } & 0.07 & 0.23 & 0.09 & NA & \\
\hline \multirow{5}{*}{ Clearfill S3 } & \multirow{2}{*}{ Un-scaled } & Count & 8 & 2 & 0 & 0 & 10 \\
\hline & & $\%$ & $80.0 \%$ & $20.0 \%$ & $0.0 \%$ & $0.0 \%$ & $100.0 \%$ \\
\hline & \multirow{2}{*}{ Scaled } & Count & 2 & 6 & 2 & 0 & 10 \\
\hline & & $\%$ & $20.0 \%$ & $60.0 \%$ & $20.0 \%$ & $0.0 \%$ & $100.0 \%$ \\
\hline & \multicolumn{2}{|c|}{ Chi-Square, P-value } & 0.02 & 0.06 & NA & NA & \\
\hline \multirow{5}{*}{ Optibond } & \multirow{2}{*}{ Un-scaled } & Count & 5 & 4 & 1 & 0 & 10 \\
\hline & & $\%$ & $50.0 \%$ & $40.0 \%$ & $10.0 \%$ & $0.0 \%$ & $100.0 \%$ \\
\hline & \multirow{2}{*}{ Scaled } & Count & 2 & 3 & 3 & 2 & 10 \\
\hline & & $\%$ & $20.0 \%$ & $30.0 \%$ & $30.0 \%$ & $20.0 \%$ & $100.0 \%$ \\
\hline & \multicolumn{2}{|c|}{ Chi-Square, $\mathrm{P}$ - value } & 0.07 & 0.09 & NA & NA & \\
\hline
\end{tabular}

NA- Not applicable, 0: no dye penetration, 1: Dye penetration along the cavity wall, but less than half of cavity depth, 2: Dye penetration along the cavity wall, more than half of the cavity depth, 3: Dye penetration spreading to and along the axial wall.

TABLE (3): Comparison of mean rank microleakage score between unscaled and scaled group.

\begin{tabular}{|c|c|c|c|c|}
\hline Treatment & Adhesive & $\mathbf{N}$ & Mean Rank & $\begin{array}{c}\text { Kruskal- } \\
\text { Wallis, } \\
\text { P- value }\end{array}$ \\
\hline \multirow{3}{*}{ Un-scaled } & Fuji Bond Lc & 10 & 8.10 & \multirow{3}{*}{0.217} \\
\hline & Clearfill S3 & 10 & 7.30 & \\
\hline & Optibond & 10 & 8.05 & \\
\hline \multirow{3}{*}{ Scaled } & Fuji Bond Lc & 10 & 12.90 & \multirow{3}{*}{0.251} \\
\hline & Clearfill S3 & 10 & 13.70 & \\
\hline & Optibond & 10 & 12.95 & \\
\hline
\end{tabular}

TABLE (4): Comparison of mean rank microleakage score between different adhesive group.

\begin{tabular}{|c|c|c|c|c|c|}
\hline Adhesive & Treatment & $\mathbf{N}$ & $\begin{array}{l}\text { Mean } \\
\text { Rank }\end{array}$ & $\begin{array}{l}\text { Sum of } \\
\text { Ranks }\end{array}$ & $P$-value \\
\hline \multirow{2}{*}{$\begin{array}{c}\text { Fuji Bond } \\
\text { Lc }\end{array}$} & Un-scaled & 10 & 8.10 & 81.00 & \multirow{2}{*}{0.06} \\
\hline & Scaled & 10 & 12.90 & 129.00 & \\
\hline \multirow{2}{*}{ Clearfill S3 } & Un-scaled & 10 & 7.30 & 73.00 & \multirow{2}{*}{0.007} \\
\hline & Scaled & 10 & 13.70 & 137.00 & \\
\hline \multirow{2}{*}{ Optibond } & Un-scaled & 10 & 8.05 & 80.50 & \multirow{2}{*}{0.06} \\
\hline & Scaled & 10 & 12.95 & 129.50 & \\
\hline
\end{tabular}




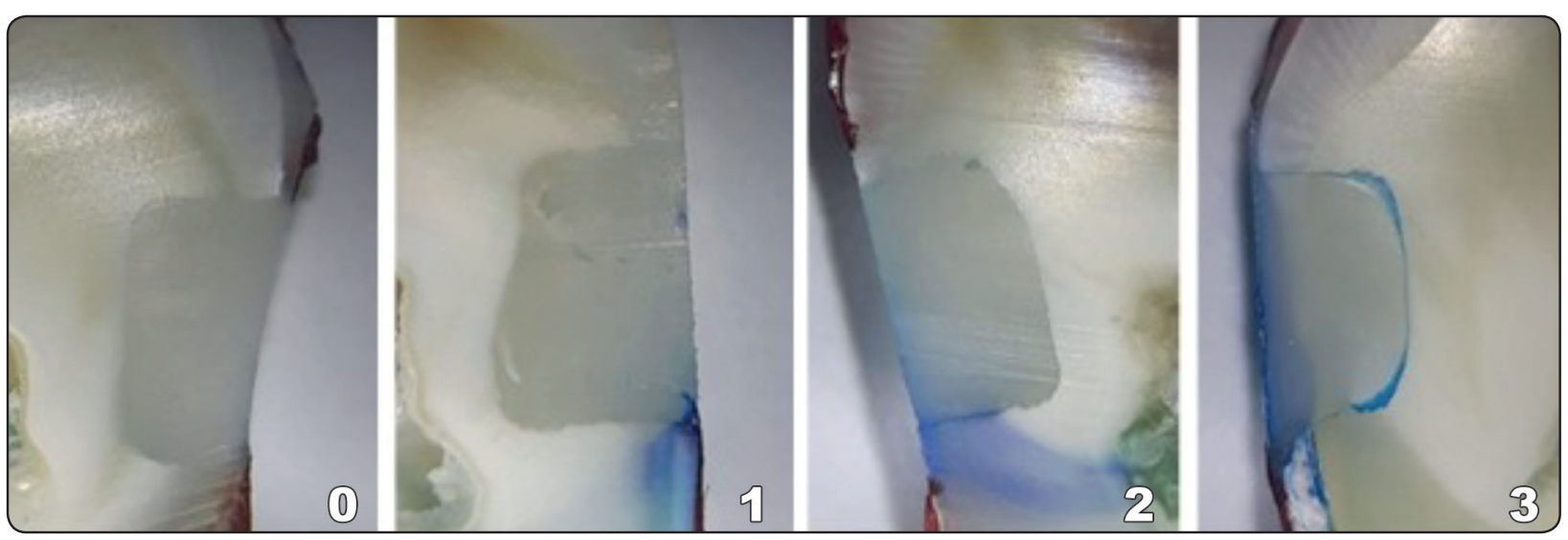

Fig. (1): Representative sectioned specimens with different microleakage scores from 0-3.

\section{DISCUSSION}

Proper bonding between the restorative material and tooth structure is influenced by the nature of dentine surface, properties of the used restorative material, intra-oral conditions and the occlusal forces . In addition, the type of applied bonding (adhesive) system plays an important role in providing and maintaining a successful restoration. ${ }^{17,18}$

The current study attempted to mimic the clinical conditions to investigate how ultrasonic scaling can influence the tooth-composite bond in the presence of three different types of adhesives, including GI, SE and ER systems, and microleakage was considered as the parameter for evaluation. This parameter was specifically selected due to its important impact on the success of composite restorations, as microleakage may lead to marginal staining and recurrent caries. ${ }^{19}$

Analyzing results of the present study displayed insignificant differences of microleakage scores between the three types of adhesives under investigation. Comparing the effect of ultrasonic scaling among each group separately showed that scaling caused a significant increase in microleakage scores only in the presence of self-etch (ClearfillS3) adhesive. On the other hand, both OptiBond FL "etch and rinse" adhesive and Fuji Bond LC Glass ionomer adhesive showed insignificant change in mi- croleakage scores after exposure to the ultrasonic scaling. These results indicate better performance of both the ER and GI adhesives in resisting microleakage, even under thermal and mechanical stresses caused by thermocycling and ultrasonic scaling. The current results can be justified in terms of the nature of the tested adhesives. Clearfill S3 Bond was presented to the market as arecent "ultramild" self-etch adhesive. This group of adhesives is characterized by lower acidity $(\mathrm{pH}>2.5$ ), which tends to produce partial demineralization and a thinner hybrid layer than that produced by total etch systems. ${ }^{20-22}$ The results of current study may indicate that the thin hybrid layer produced by SE adhesive (Clearfill S3 Bond)was not enough to maintain proper bonding in presence of the stresses generated by ultrasonic scaling. On the other hand, OptiBond FL, is a conventional etch and rinse (ER) adhesive which totally demineralizes dentine surface and opens dentinal tubules. ${ }^{6,23}$ According to the current results, this method proved more durable bond and less microleakage after exposure to thermocycling and scaling. The resistance of GI adhesive (Fuji BondLC) to microleakage can be attributed to its ability to bond chemically to tooth structure and micro-mechanically to composites. In addition, the unique flexible nature of the material allows for expansion and contraction of the resin restorative without opening the cavo-surface margins. ${ }^{10,24,25}$ 
Current study results came in agreement with El Sayed et al. ${ }^{16}$ who compared "self-etch" to "etch and rinse" systems, as they concluded that "etch and rinse"adhesive deserved its position as the gold standard bonding agent, as it showed less microleakage. On the contrary, Gupta et al. ${ }^{26}$, evaluated microleakage with different systems, and they came to the conclusion that one step self-etch adhesives revealed less microleakage than total etch ones. Authors of the current research found very less publications that study the effect of scaling on bonded composite restorations. One research by Bagis et al..$^{27}$, tested the effect of ultrasonic agitation on bond strength of self-etching adhesives to dentin. The result showed ultrasonic agitation had no effect on bonding performance of the SE adhesive. As bond strength is considered as a major factor that affects microleakage, so these results are considered on the contrary of those gained by our study. This disagreement may be explained in terms of different methodology or mismatching of the commercial brands of the tested adhesives.

A more recent study by Goldstein et al. ${ }^{15}$, investigated microleakage around ClassV composite restorations with ultrasonic scaling and sonic toothbrushing, in presence of total etch adhesive only. Author concluded that microleakage at cementumcomposite interface increased with ultrasonic scaling, but not with sonic toothbrushing. Unfortunately, no previous data were available to compare GI adhesive to SE and ER adhesives, regarding their ability to resist microleakage after ultrasonic scaling. Thus, more studies are required in this instance.

\section{CONCLUSION AND RECOMMENDATION}

Under limitations and conditions of the present in-vitro study, the following conclusions could be made:

1. In the un-scaled condition, non of the tested adhesives resulted in a microleakage-free restoration.

2. Ultrasonic scaling significantly increased microleakage in restorations bonded with the self-etch adhesive. However, glass-ionomer and etch \& rinse adhesives revealed more resistance to the effect of ultrasonic scaling.

3. Clinical trials are recommended to assess the performance of these adhesive systems under different oral conditions.

\section{REFERENCES}

1. Darabi F, Tayefeh-Davalloo R, Tavangar SM, Naser-Alavi F, Boorboo-Shirazi M. The effect of composite resin preheating on marginal adaptation of class II restorations. J Clinical Experimental Dent. 2020;12: e682-e687.

2. Kemaloglu H, Pamir T, Tezel H. A 3-year randomized clinical trial evaluating two different bonded posterior restorations: Amalgam versus resin composite. Eur J Dent. 2016;10:16-22.

3. Sarrett DC. Prediction of clinical outcomes of a restoration based on in vivo marginal quality evaluation. J Adhes Dent. 2007;9:117-20.

4. Atoui JA, Chinelatti MA, Palma-Dibb RG, Corona SA. Microleakage in conservative cavities varying the preparation method and surface treatment. J Appl Oral Sci.2010;18:421-5.

5. Martins GC, Sánchez-Ayala A, D'Alpino PHP, Calixto AL, Gomes JC, Gomes OMM. Interfacial integrity of bonded restorations with self-etching adhesives: Water storage and thermo-mechanical cycling. Eur J Dent. 2012;6:169-177.

6. Silva e Souza MH Jr, Carneiro KG, Lobato MF, Silva e Souza PA, Góes MF. Adhesive systems: important aspects related to their composition and clinical use. J Appl Oral Sci.2010;18:207-14.

7. Ren L, Li M, Pan Y, Meng X. Influence of Polishing Methods on the Bonding Effectiveness and Durability of Different Resin Cements to Dentin. Biomed Res Int. 2018: 9189354.

8. Abad-Coronel C, Naranjo B, Valdiviezo P. Adhesive Systems Used in Indirect Restorations Cementation: Review of the Literature. Dent J (Basel). 2019;7:71.

9. van Meerbeek B, De Munck J, Yoshida Y, Inoue S, Vargas M, Vijay P, Van Landuyt K, et al. Buonocore Memorial Lectured adhesion to enamel and dentin: current status and future challenges. Oper Dent.2003;28 215-35.

10. Geerts SO, Seidel L, Albert AI, Gueders AM . Microleakage after Thermocycling of Three Self-Etch Adhesives under Resin-Modified Glass-Ionomer Cement Restorations. Int J Dent. 2010:728453. 
11. Tyas MJ,Burrow MF. Clinical evaluation of a resin modified glass ionomer adhesive system: Results at five years. Oper Dent.2002;27:438-41.

12. Van Ende A, De Munck J, Mine A, Lambrechts P, Van Meerbeek B. Does a low-shrinking composite induce less stress at the adhesive interface? Dent Mater. 2010;26:215-22.

13. Kakaboura A, Palaghias G, Eliades G. Setting characteristics and cavity adaptation of low-shrinking resin composites. Dent Mater.2009;25:1509-16.

14. Oduncuoğlu BF, Yamanel K, Koçak ZS. In Vitro Evaluation of Direct and Indirect Effects of Sonic and Ultrasonic Instrumentations on the Shear Bond Strength of Orthodontic Brackets. Turk J Orthod. 2020;33:37-42.

15. Goldstein RE, Lamba S, Lawson NC, Beck P, Oster RA, Burgess JO. Microleakage around Class V Composite Restorations after Ultrasonic Scaling and Sonic Toothbrushing around their Margin. J Esthet Restor Dent. 2017;29: 41-48.

16. El Sayed HY, Abdalla AI, Shalby ME. Marginal microleakage of composite resin restorations bonded by desensitizing one step self etch adhesive. Tanta Dental Journal. 2014;11:180-188.

17. Kermanshah H, Yasini E, Hoseinifar R. Effect of cyclic loading on microleakage of silorane based composite compared with low shrinkage methacrylate-based composites. Dent Res J (Isfahan).2016;13:264-271.

18. Sultan A, Moorthy A, Fleming GJ. The adhesive potential of dentin bonding systems assessed using cuspal deflection measurements and cervical microleakage scores. Dent Mater.2014;30:1154-60.

19. Balkaya H, Arslan S, Pala K. A randomized, prospective clinical study evaluating effectiveness of a bulk-fill composite resin, a conventional composite resin and a reinforced glass ionomer in Class II cavities: one-year results. J Appl Oral Sci. 2019;27 :e20180678.

20. Yoshida Y, Nagakane K, Fukuda R, et al. Comparative study on adhesive performance of functional monomers. $\mathrm{J}$ Dent Res.2004;83:454-8.

21. Van Meerbeek B, Yoshihara K, Yoshida Y, Mine A, De Munck J, Van Landuyt KL. State of the art of self etch adhesives. Dent Mater. 2011;27:17-28.

22. Carvalho RM, Tjäderhane L, Manso AP, Carrilho MR, Carvalho CA. Dentin as a bonding substrate. Endod Topics. 2012;21:62-88.

23. Abu-Nawareg MM, Zidan AZ, Zhou J, et al. Adhesive sealing of dentin surfaces in vitro: A review. Am J Dent. 2015;28:321-332.

24. Oglakci B, Kazak M, Donmez N, Dalkilic EE, Koymen SS. The use of a liner under different bulk-fill resin composites: 3D GAP formation analysis by $\mathrm{x}$-ray microcomputed tomography. J Appl Oral Sci. 2020;28:e20190042.

25. Inoue $\mathrm{S}, \mathrm{Abe} \mathrm{Y}$, Yoshida $\mathrm{Y}$, et al. Effect of conditioner on bond strength of glass-ionomer adhesive to dentin/enamel with and without the smear layer interposition. Oper Dent. 2004; 29 : 685-692.

26. Gupta A, TaTavane P, Gupta PK, et al. Evaluation of Microleakage with Total Etch, Self Etch and Universal Adhesive Systems in Class V Restorations: An In vitro Study. J Clin Diagn Res. 2017;11:ZC53-ZC56.

27. Bagis B, Turkarslan S, Tezvergil-Mutluay A, Uctasli S, Vallittu PK, Lassila LV. Effect of ultrasonic agitation on bond strength of self-etching adhesives to dentin. J Adhes Dent.2008;10:441-5. 